\title{
Quasimorphisms and laws
}

\author{
DANNY CALEgARI
}

Stable commutator length vanishes in any group that obeys a law.

20E10; 20F65, 57M07, 20J05

If $G$ is a group and $g$ is an element of the commutator subgroup $[G, G]$, the commutator length of $g$, denoted $\operatorname{cl}(g)$, is the least number of commutators in $G$ whose product is $g$. The stable commutator length, denoted $\operatorname{scl}(g)$, is the limit $\operatorname{scl}(g):=\lim _{n \rightarrow \infty} \operatorname{cl}\left(g^{n}\right) / n$. A group $G$ is said to obey a law if there is a free group $F$ (which may be assumed to have finite rank) and a nontrivial element $w \in F$ so that for every homomorphism $\rho: F \rightarrow G$, we have $\rho(w)=$ id. For example, abelian (or, more generally, nilpotent or solvable) groups obey laws. The free Burnside groups $B(m, n)$ with $m \geq 2$ generators and odd exponents $n \geq 665$ are perhaps the best known examples of non-amenable groups that obey laws; see for example Adyan [1].

The point of this note is to prove the following:

Main Theorem Let $G$ be a group that obeys a law. Then $\operatorname{scl}(g)=0$ for every $g \in[G, G]$.

The proof is very short, given some basic facts about stable commutator length, which we recall for the convenience of the reader. A basic reference is Bavard's paper [2] or the author's monograph [3], especially Chapter 2.

Definition 1 A homogeneous quasimorphism on a group $G$ is a function $\phi: G \rightarrow \mathbb{R}$ that restricts to a homomorphism on every cyclic subgroup, and for which there is a least number $D(\phi) \geq 0$ (called the defect) so that for any $g, h \in G$ there is an inequality $|\phi(g h)-\phi(g)-\phi(h)| \leq D(\phi)$.

The defect satisfies the following formula:

Lemma 2 [2, Lemma 3.6] or [3, Lemma 2.24] Let $\phi$ be a homogeneous quasimorphism. Then there is an equality

$$
\sup _{g, h \in G}|\phi([g, h])|=D(\phi) .
$$


Bavard duality (see [2] or [3, Theorem 2.70]) says that for any $g \in[G, G]$, there is an equality $\operatorname{scl}(g)=\sup _{\phi} \phi(g) / 2 D(\phi)$ where the supremum is taken over all homogeneous quasimorphisms $\phi$ with nonzero defect. In particular, scl is nontrivial on $G$ if and only if $G$ admits a homogeneous quasimorphism with nonzero defect.

On the other hand, there is a topological formula for scl. Let $X$ be a space with $\pi_{1}(X)=G$, and let $\gamma: S^{1} \rightarrow X$ be a free homotopy class representing the conjugacy class of $g \in G$. If $\Sigma$ is a compact, oriented surface without sphere or disk components, a map $f: \Sigma \rightarrow X$ is admissible if the map $\partial f: \partial \Sigma \rightarrow X$ can be factorized as $\partial \Sigma \stackrel{d}{\rightarrow}$ $S^{1} \stackrel{\gamma}{\rightarrow} X$. For an admissible map, define $n(\Sigma)$ by the equality $d_{*}[\partial \Sigma]=n(\Sigma)\left[S^{1}\right]$ in $H_{1}$; i.e. $n(\Sigma)$ is the degree with which $\partial \Sigma$ wraps around $\gamma$. By reversing the orientation of $\Sigma$ if necessary, we assume $n(\Sigma) \geq 0$. With this notation, one has the following formula:

Lemma 3 [3, Proposition 2.10] With notation as above,

$$
\operatorname{scl}(g)=\inf _{\Sigma} \frac{-\chi(\Sigma)}{2 n(\Sigma)}
$$

where $\chi$ denotes Euler characteristic, and the infimum is taken over all compact, oriented surfaces and all admissible maps.

Notice that both $\chi(\cdot)$ and $n(\cdot)$ are multiplicative under finite covers.

Proof of the Main Theorem Suppose that $G$ obeys a law. Then there is a free group $F$ and a nontrivial word $w \in F$ so that any homomorphism from $F$ to $G$ sends $w$ to id. Let $F_{2}$ be free on generators $x, y$. We can embed $F$ in $F_{2}$, and express $w$ as a word $v$ in the generators $x, y$. Hence any homomorphism from $F_{2}$ to $G$ sends $v$ to id.

Let $X$ be a space with $\pi_{1}(X)=G$. Let $\Sigma$ be a once-punctured torus. We choose generators for $\pi_{1}(\Sigma)$, and identify this group with $F_{2}=\langle x, y\rangle$. Let $\alpha$ be a loop on $\Sigma$ whose free homotopy class represents the conjugacy class of $v$. Then any continuous map $f: \Sigma \rightarrow X$ sends $\alpha$ to a null-homotopic loop.

Now suppose contrary to the theorem that scl does not vanish on $[G, G]$. By Bavard duality there is a homogeneous quasimorphism $\phi$ with nonzero defect. Scale $\phi$ to have $D(\phi)=1$. Then by Lemma 2, for any $\epsilon>0$ there are elements $g, h$ in $G$ with $\phi([g, h]) \geq 1-\epsilon$, and consequently $\operatorname{scl}([g, h]) \geq 1 / 2-\epsilon / 2$ by Bavard duality.

Let $\gamma: S^{1} \rightarrow X$ be a loop representing the conjugacy class of $[g, h]$. There is a map $f: \Sigma \rightarrow X$ whose boundary represents the free homotopy class of $\gamma$. As above, the loop $\alpha$ on $\Sigma$ maps to a null-homotopic loop in $X$. By Scott [4], there is a finite cover 
$\widetilde{\Sigma}$ of $\Sigma$ of degree $d$ (depending on $\alpha$ ), so that some lift $\tilde{\alpha}$ of $\alpha$ is homotopic to an embedded loop $\alpha^{\prime}$. Composing the covering map with $f$ gives a map $\tilde{f}: \widetilde{\Sigma} \rightarrow X$ for which $\widetilde{f}\left(\alpha^{\prime}\right)$ is null-homotopic in $X$. Since $\alpha^{\prime}$ is embedded, we can compress $\widetilde{\Sigma}$ along $\alpha^{\prime}$ to produce a new surface $\Sigma^{\prime}$ mapping to $X$ by $f^{\prime}$. The map $f^{\prime}$ is admissible for $\gamma$, and satisfies $n\left(\Sigma^{\prime}\right)=d$. Moreover, $\chi(\widetilde{\Sigma})=-d$, and $\chi\left(\Sigma^{\prime}\right)=2-d$. Consequently, by Lemma 3 , we have $\operatorname{scl}([g, h]) \leq 1 / 2-1 / d$.

Since $d$ is fixed (depending only on the law satisfied by $G$ ) but $\epsilon$ is arbitrary, we obtain a contradiction. Hence scl vanishes identically on $[G, G]$, as claimed.

Remark 4 The statement of the Main Theorem may be rephrased positively as saying that if scl is nonzero on $G$, then for any positive integer $n$, there are homomorphisms $F_{2} \rightarrow G$ which are injective on the ball of radius $n$.

If $w$ is a word in a free group $F$, define a $w$-word in $G$ to be the image of $w$ under a homomorphism $F \rightarrow G$. Let $G(w)$ be the subgroup of $G$ generated by $w$-words. The $w$-length of $g \in G(w)$, denoted $1(g \mid w)$, is the smallest number of $w$-words and their inverses whose product is $g$ (commutator length is the case $w=x y x^{-1} y^{-1} \in\langle x, y\rangle$ ), and the stable $w$-length, denoted $\operatorname{sl}(g \mid w)$ is $\operatorname{sl}(g \mid w):=\lim _{n \rightarrow \infty} 1\left(g^{n} \mid w\right) / n$.

Question 5 Is there an example of a group that obeys a law, but for which $\operatorname{sl}(\cdot \mid w)$ is nontrivial for some $w$ ?

This work was partially supported by NSF grant DMS 0707130. I would like to thank the anonymous referee for helpful comments on an earlier draft.

\section{References}

[1] S I Adyan, Random walks on free periodic groups, Izv. Akad. Nauk SSSR Ser. Mat. 46 (1982) 1139-1149, 1343 MR682486

[2] C Bavard, Longueur stable des commutateurs, Enseign. Math. (2) 37 (1991) 109-150 MR1115747

[3] D Calegari, scl, MSJ Memoirs 20, Mathematical Society of Japan, Tokyo (2009) MR2527432

[4] P Scott, Subgroups of surface groups are almost geometric, J. London Math. Soc. (2) 17 (1978) 555-565 MR0494062

Department of Mathematics, Caltech, Pasadena CA 91125, USA

dannyc@caltech.edu

http://www.its. caltech.edu/ dannyc

Received: 4 October 2009 